\title{
A Study of Connection Management Approaches for an impairment-aware Optical Control Plane
}

\author{
Elio Salvadori ${ }^{1}$, Yabin $\mathrm{Ye}^{1}$, Andrea Zanardi ${ }^{1}$, Hagen Woesner $^{1}$, Matteo Carcagnì ${ }^{1}$ \\ Gabriele Galimberti ${ }^{2}$, Giovanni Martinelli ${ }^{2}$, Alberto Tanzi ${ }^{2}$, Domenico La Fauci ${ }^{2}$ \\ ${ }^{1}$ Create-Net, Via Solteri 38, Trento, Italy, 38100 \\ \{elio.salvadori, yabin.ye, andrea.zanardi, hagen.woesner, matteo.carcagni\} @ create-net.org \\ ${ }^{2}$ Cisco Optical Networking Group, Via Philips, 12, 20052 - Monza (MI), Italy \\ \{ggalimbe, giomarti, atanzi, dlafauci\} @ cisco.com
}

\begin{abstract}
Transparent optical networks need novel connection management approaches to take into account the presence of physical impairments in lightpath provisioning. Two main schemes are emerging from literature when considering how to introduce impairment-aware mechanisms in a distributed optical control plane like GMPLS. A well-known approach is based on extending the routing protocol to compute an optically-feasible light-path. Lately, a new approach is emerging which keeps the routing protocol unmodified while leveraging on signaling protocol extensions to find the proper lightpath for the incoming connection request. The aim of this paper is to prove that the signaling-based approach has several advantages compared to the routing-based one, in term of scalability and robustness especially when link information changes are frequent in the network. Simulation results show that a signaling-based approach is much more robust to inaccurate information about network status, therefore it is a suitable approach for considering physical impairments in dynamic optical networks.
\end{abstract}

Keywords: Generalized Multi-Protocol Label Switching (GMPLS); Optical Control Plane (OCP); Wavelength Division Multiplexing (WDM); Routing and Wavelength Assignment (RWA); Physical Impairments

\section{Introduction}

Generalized Multi-Protocol Label Switching (GMPLS) has been proposed for managing the control plane in Wavelength Division Multiplexed (WDM) optical networks [1]. The routing protocol used in GMPLS is open shortest path first with traffic engineering extensions (OSPF-TE) [2], which has two main objectives: i) to provide the nodes with a dynamic and more exact view on network status (capacity, load, congestion state and other link attributes) and ii) to enable constrained-based routing (CBR) to be run in each node, in order to perform intelligent path computation (explicit route determination). Resource Reservation with traffic engineering extensions (RSVP-TE) [3] is the signaling protocol often considered to establish the 
path whose explicit route has been calculated through some CBR mechanism, by instantiating a label forwarding state along the path itself.

Standard GMPLS does not take into account the physical impairments of real optical networks. That means it assumes an ideal network where optical signals are transmitted from source to destination nodes without degradation. While this assumption is largely correct for optical networks with $\mathrm{O} / \mathrm{E} / \mathrm{O}$ conversion in each node, in upcoming photonic networks employing purely optical crossconnects (OXC), due to transmission impairments (insertion losses, amplified spontaneous emission (ASE) noises, polarization mode dispersion (PMD), chromatic dispersion (CD), crosstalk, etc.) accumulation, the signal significantly degrades when it travels through the lightpath. Most of the currently used route computation algorithms would perform badly, if not fail, in establishing a working lightpath since they are not aware of the specific physical layer constraints and the path selected would possibly lead to unacceptably high bit-error rates (BER) [4-8].

There are two main approaches to introduce impairment-awareness in a GMPLSbased distributed optical control plane [9]. The first approach (routing-based) introduces additional physical information into the routing protocol, i.e. OSPF-TE [10-11]. By flooding Link State Advertisements (LSAs), all the nodes populate their Traffic Engineering Database (TED) information which gives them a view of the whole network. Each node can then find the right route for a connection request while standard RSVP-TE signaling is used for lightpath establishment.

The second approach (signaling-based) extends the RSVP-TE protocol instead [12]. Each node can select a route based on standard OSPF-TE protocol without knowledge of physical impairments and then the feasibility of the computed optical path is evaluated on a hop-by-hop basis in each node along the path during lightpath set-up. In this case RSVP-TE messages are extended to include physical information.

An initial theoretical assessment of these two approaches is reported in [9], where the authors reflect on the main limitations of the routing-based approach, such as TED inconsistency as well as scalability and stability problems when the link information changes are frequent [11]. Another limitation of this approach is the impact on the NE's Control Unit CPU, whose load is heavily stressed by the complexity of the multi-constrained path computation algorithm required for guaranteeing both optimal network performance and sufficient quality of the optical signal. On the other hand, the signaling-based approach better handles frequent changes of the physical parameters, and no global flooding of physical information is required, thereby minimizing scalability problems. Furthermore since no complex path computation algorithms are used, the load on the NE's CU is minimized. However, the main drawbacks of this approach are: a bigger set-up delay due to an increased number of set-up attempts as well as a sub-optimal resource allocation due to the impairment-unaware route computation algorithm used.

In this paper, for the first time to our knowledge, an in-depth comparison of these two approaches is provided through an extensive simulation activity. In particular, the increased robustness to imprecise network state information as well as much better scalability of the signaling-based approach compared to the routing-based is demonstrated, while showing reduced impact on the set-up delay. Notice that the set of impairments considered in this work has been restricted to the linear ones to limit the complexity of the problem we have been studying; however according to the 
obtained results, including non-linear impairments would have further highlighted the limits of a routing-based approach compared to a signaling-based one.

Section 2 of this paper provides details of the two optical control plane (OCP) approaches considered. In Section 3 we present and discuss illustrative numerical examples to evaluate the two approaches. Section 4 concludes this paper. The models for the physical impairments used in our simulations are presented in the Appendix A.

\section{Impairment-Aware Optical Control Plane Architectural Options}

In the following the two approaches previously introduced are described in more detail.

\subsection{Signaling-based OCP}

In this approach, no extensions to the routing protocols to describe transmission impairments and wavelength availability are introduced. Instead, each node has only a local knowledge of the physical parameters related to its adjacent links. In this architectural option the verification of the feasibility of a lightpath is entirely delegated to the signaling phase. The mechanism considered in this paper is very close to the one proposed in [12], but with respect to this a realistic mathematical model for evaluating several linear transmission impairments considered has been used (see Appendix A for more details) instead of a generic additive linear parameter.

Each time a node receives a request to set up a connection, a local path computation element will compute a route to the destination node. Note that this route may not be feasible from the photonic domain point of view. If established, it would result in an unacceptably high BER, violate service level agreements (SLA) while at the same time reserve capacity in the network. In this approach some extensions are therefore introduced in the signaling messages (e.g. RSVP-TE) to collect signal impairments characterizing the traversed links from the ingress to the egress node. In particular, the idea is to include into a Path message one or more fields containing information about the optical signal level (e.g. signal power, optical signal to noise ratio OSNR) and accumulated impairments (e.g. CD, Crosstalk, PMD) per wavelength to be set up. This information is updated at every traversed node together with the set of available wavelengths by leveraging the local knowledge of the physical parameters available in the node itself. The egress node will evaluate the feasibility of a suggested wavelength (label) by evaluating the transmission quality according to the client interface terminating the lightpath (in terms of BER, OSNR sensitivity, $\mathrm{CD}$ tolerance, etc.). If at least one of the suggested wavelengths is feasible, the egress node will send the Resv message back to the ingress selecting the wavelength in order to set up the lightpath. If more than one are feasible, the lowerorder one is selected according to a First-Fit policy. If not, a ResvErr message must be sent back and a new route to establish the connection request must be calculated.

Compared to [12], which is performing only up to two re-attempts, we have been allowing the ingress node to perform up to $\mathrm{K}$ attempts to set-up a lightpath. A KCSPF path computation algorithm has been implemented in the simulator, which 
provides the ingress node with up to $\mathrm{K}$ possible paths to set up a lightpath to the egress node. While a high $\mathrm{K}$ increases the probability to successfully set-up a lightpath request, it also dangerously increases the lightpath set-up delay. The proper value of $\mathrm{K}$ should therefore be carefully selected depending on the network topology and on the size of the transparent domain. Anyway, simulation results demonstrate that good performance can be generally obtained even with low values of $\mathrm{K}$.

\subsection{Routing-based OCP}

In this case, each node must have an overall view of the resource utilization and the physical parameters in the network, maintained through a TED available locally. This means that some extensions to the routing protocol (i.e. to LSA messages of OSPFTE protocol) must be implemented to carry wavelength usage and transmission impairments information in addition to TE attributes. Each time a node receives an incoming connection request, a local path computation element will compute a route through a constrained-based routing algorithm which takes into account the wavelength availability as well as the physical impairments. The ICBR algorithm used in this paper is very close to the one proposed in [8], with two modules, the network-layer module ${ }^{1}$ which computes a set of candidate lightpaths for the incoming request, among which one of them is selected through a physical-layer module responsible for checking its optical feasibility. However, with respect to [8] which was focusing on the performance of the ICBR algorithm only, in this paper the effect of the OSPF protocol extension is studied. Furthermore, more transmission impairments have been considered.

It is important to note that for the mathematical models considered in our simulations the only dynamic information that needs to be updated via LSA messages is the wavelength availability per link. All other parameters considered in Appendix A are of static nature or function of the number of wavelengths and can thus be pre-loaded onto each NE during the provisioning phase. Once a node receives the specific wavelength availability information per link, it can compute the optical feasibility through its physical-layer module implementing the equations described in Appendix $\mathrm{A}^{2}$. The optically feasible computed path would then be set up through standard RSVP-TE selecting one of the available wavelengths according to a First-Fit policy.

In this approach, the TED instance available locally inside each node is supposed to be the same in the all network. However, due to misalignment and routing protocol convergence time in case of network changes (topology, link availability, etc), this database cannot be $100 \%$ guaranteed to reflect the real network status. It is worth to note that in the case of an extended LSA any change in the number of available wavelengths in some fiber link will generate new update messages thus frequently misaligning for some time interval the TED instances in the network nodes. Another disadvantage of this approach is that in the case of a heterogeneous multi-vendor

${ }^{1}$ Note that for the implementation of the network-layer module we have been re-using the KCSPF path computation algorithm used in the Signaling-based OCP, with a very high value for $\mathrm{K}$

2 Note that this is a strong assumption that is reasonable only in the case of a homogeneous network scenario (nodes are provided by the same vendor). 
network the TED will potentially need to store information for different kinds of nodes and links. For a change or update in the network, a significant amount of parameters need to be updated, which can cause stability and scalability problems.

\section{Numerical Results and Discussion}

In this section, the Signaling based-OCP and Routing based-OCP are evaluated through numerical simulations performed with an extended version of the GLASS simulator [14]. In the simulations, typical regular network (Mesh-Torus, Fig. 1(a)), real network (AT\&T network [15], Fig. 1(b)) and quasi-regular network (Grid, Fig. (c)) have been studied. Both Mesh-Torus and Grid networks have fiber links $80 \mathrm{~km}$ long, so only pre- and booster optical amplifiers are used inside each node, while no in-line optical amplifier are used per link. For simulation purposes, the AT\&T topology has been scaled down as well (by a factor of 1:23) to avoid in-line optical amplifiers in all fiber links. In all the networks, nodes are connected through pairs of uni-directional WDM links with 32 wavelengths each; all connections are bidirectional using the same wavelength in both directions.

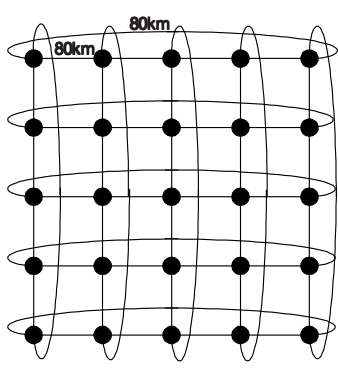

(a)

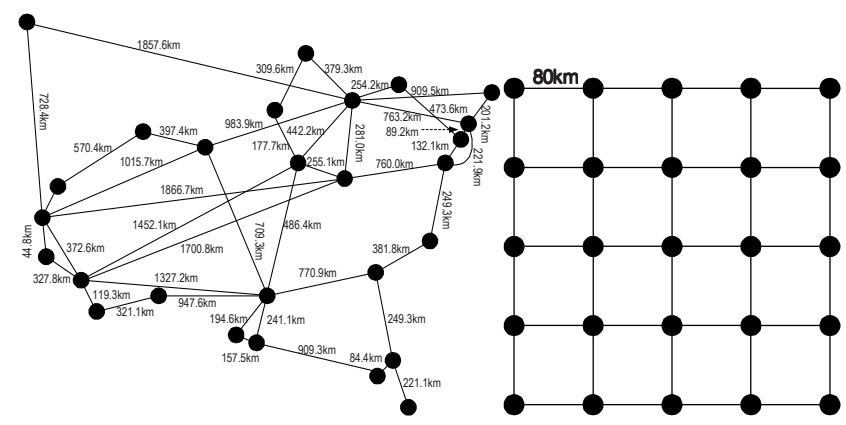

(b)

(c)

Fig. 1. The network topologies used for simulations (a) $5 \times 5$ Mesh-Torus network (b) AT\&T network (c) $5 \times 5$ Grid network

The traffic scenario in the simulation consists of a classical traffic model based on lightpath requests with Poisson arrivals average rate $(1 / \mu$ per second) and average exponential duration with $v$ seconds. The traffic requests are uniformly distributed among all the nodes. The traffic load is defined as the average network resource (link wavelength) usage computed as:

$$
\frac{\bar{N}_{c} \times \bar{L}_{c}}{M \times W} \times 100 \%
$$

where $\bar{N}_{c}$ is the average number of active connections and equals $v / \mu, \bar{L}_{c}$ is the average number of hops in the network (considering only shortest paths between nodes), $M$ is the number of links and $W$ is the number of wavelengths in the network. 
In the simulation, route selection is performed in the source node using a modified breadth-first search algorithm that iteratively computes the first $\mathrm{K}$ distinct paths satisfying the constraints on available link resources. In routing-based OCP only the first selected path satisfying the wavelength continuity constraint and optically feasible is returned ( $\mathrm{K}$ is virtually infinite).

The purpose of the performed simulations is to compare the signaling-based to the routing-based OCP approach in GMPLS. We first compare the tolerance of each OCP approach to the inaccurate TED information and then show how strong is the effect of the increased set up time in a signaling-based OCP architecture.

In order to study the influence of inaccurate information on both architectures, we artificially introduce an OSPF message processing delay. When each node receives a LSA from its neighbor, it will hold it for a certain number of seconds before flooding it to its neighbors. In this case, all the nodes will have outdated network status.

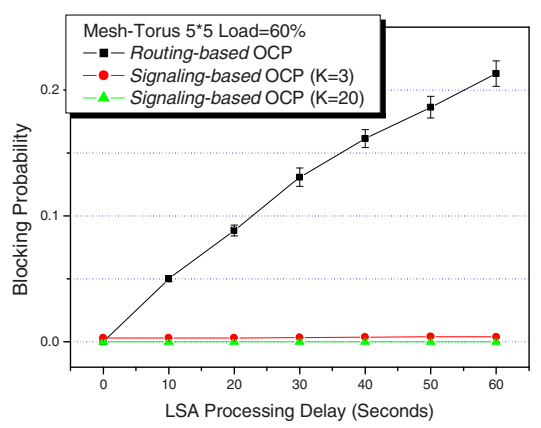

(a)

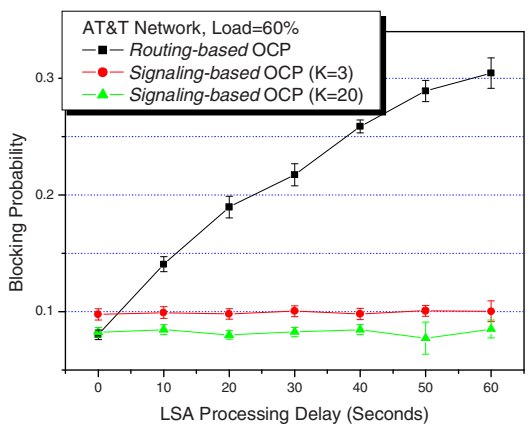

(b)

Fig. 2 signaling-based OCP vs. routing-based OCP with increasing LSA processing delay in (a)Mesh-Torus $5 \times 5$ network, (b) AT\&T network

Fig. 2 compares the blocking probability of signaling-based OCP (for both $\mathrm{K}=3,20$ ) and routing-based OCP changing with LSA processing delay in Mesh-Tours $5 \times 5$ network and AT\&T network when the traffic load is $60 \%$ and the average request inter-arrival time $\mu$ is 2 seconds. This interarrival frequency is supposed to model a dynamic lightpath requests due to a grooming policy requesting new lightpaths each time highly dynamic IP traffic is crossing a 50\% occupation threshold per established lightpath, which is a common assumption for most Tier-1 ISPs. The LSA Processing Delay has been overestimated to better show its effect on the performance of both OCP architectures. When there is no LSA processing delay, routing-based OCP has similar blocking probability performance to the signaling-based $\mathrm{OCP}$ with $\mathrm{K}=20$ and it is slightly better than that with $\mathrm{K}=3$ (our simulations proved this result is valid for all traffic loads and on different network topologies, but we do not include them here for space reasons). However, by increasing the LSA processing delay, the blocking probability of routing-based OCP architecture increases quickly, while for the signaling-based OCP architecture it remains stable for both $\mathrm{K}=3$ and 20 . A signalingbased OCP is therefore more robust to inaccurate network information than routingbased OCP. 


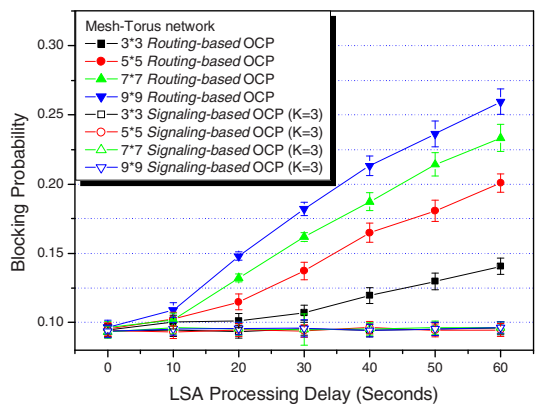

(a)

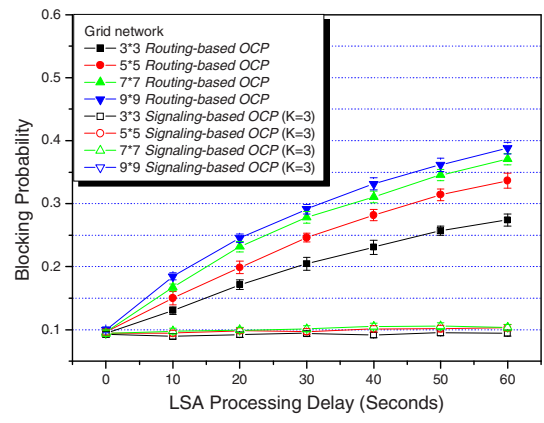

(b)

Fig. 3 - Scalability of signaling-based OCP and routing-based OCP (a) Mesh-Torus Network, (b) Grid Network

In order to check our assumption of a better scalability of the signaling-based OCP compared to the routing-based OCP architecture we have been running a set of simulations on both a regular (Mesh-Torus) and a quasi-regular (Grid) topology with increasing number of nodes. The curves of the network blocking probability changing with increasing LSA processing delay are shown in Fig. 3. The starting point for different network sizes is obtained by finding the traffic load corresponding to the same network blocking probability when there is no LSA delay. For a routing-based OCP architecture, the larger the network, the faster the blocking probability increases with the LSA delay. This is because in large networks, the TED of each node needs more time to be updated and therefore the source node has less accurate information when calculating the $\mathrm{K}$ paths. On the other hand, for signaling-based OCP architecture, the blocking probability is almost the same for different network sizes and does not change with increasing LSA delay. This means that a signaling-based OCP approach is more scalable than a routing-based OCP, as deferring in the signaling phase wavelength selection and optical feasibility verification in the signaling phase using local node information greatly reduces the influence of TED misalignments.

It has been proven from Fig.2 and Fig.3 that signaling-based $O C P$ is more tolerable to inaccurate TED information and therefore has better scalability than routing-based OCP. However, as it is stated in Section 1, its main weakness is the longer lightpath set-up time, which is proportional to the average number of attempts, defined as the number of distinct paths the source node has tried before it receives the Resv message. 


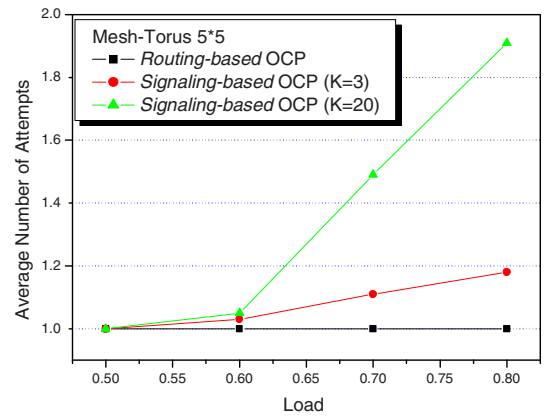

(a)

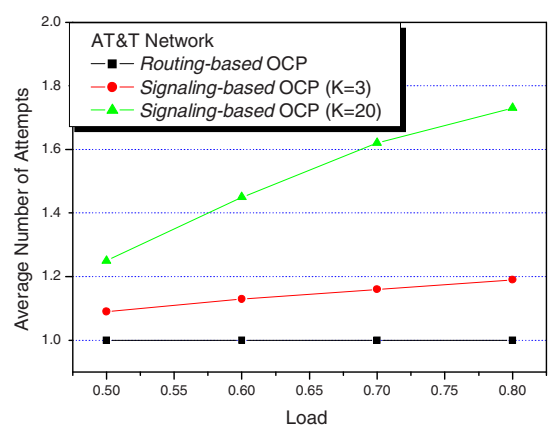

(b)

Fig. 4 Comparison of average number of attempts between signaling-based OCP and routingbased OCP in (a) Mesh-Torus $5 \times 5$ network, (b) AT\&T network

Fig. 4 shows the average number of attempts changing with the traffic load for signaling-based $\mathrm{OCP}$ and routing-based $\mathrm{OCP}$ in Mesh-Torus $5 \times 5$ network and AT\&T network. The average number of attempts is obtained by averaging number of attempts over all the successfully established connections. While the number of attempts for the routing-based OCP is always 1, the number of attempts for signalingbased OCP increases with the traffic load. However, it can be found from Fig. 4 that the average number of attempts for signaling-based OCP $(K=3)$ is not higher than $20 \%$ of the routing-based OCP. This indicates that the impact on the network performance of the reattempts performed to discover an optically feasible path is not severe, at least for low $\mathrm{K}$ values. Of course for $K=20$ this impact is stronger as the number of attempts almost doubles for high traffic load.

\section{Conclusions}

In this paper we have studied the problem of enhancing GMPLS for considering physical impairments in real optical networks. Two approaches have been compared: a routing-based OCP architecture which is extending the OSPF-TE protocol to broadcast physical impairments to all nodes and then to find the appropriate route and wavelength based on TED; a signaling-based OCP architecture which is instead extending the RSVP-TE protocol to check the feasibility of the lightpath in a hop by hop manner. These two approaches have been implemented in GLASS simulator by considering a set of linear physical impairments (Loss, ASE noise, PMD, CD and Crosstalk, etc). An extensive set of numerical simulation performed on both regular and arbitrary topologies proved that a signaling-based OCP architecture is much more robust to inaccurate network information thanks to its distributed nature, with a limited impact on the connection setup time. Furthermore this approach is also more scalable than routing-based OCP for networks with high number of nodes. 


\section{References}

1. RFC 3945 - Generalized Multi-Protocol Label Switching Architecture - October 2004

2. RFC 4203 - OSPF Extensions in Support of Generalized Multi-Protocol Label Switching October 2005

3. RFC 3473 - Generalized Multi-Protocol Label Switching (GMPLS) Signaling Resource ReserVation Protocol-Traffic Engineering (RSVP-TE) Extensions - January 2003

4. B. Mukherjee: Optical communication Networks, New York: McGraw-Hill, July 1997

5. B. Ramamurthy, D. Datta, H. Feng, J. P. Heritage, B. Mukherjee, Impact of Transmission Impairments on the Teletraffic Performance of Wavelength-routed Optical Networks, IEEE/OSA Journal of Lightwave Technology, vol. 17, no. 10, pp. 1713-1723, Oct. 1999.

6. Teck Yoong Chai, Tee Hiang Cheng, Yabin Ye, Qiang Liu, Inband Crosstalk Analysis of Optical Cross-Connect Architectures, IEEE/OSA Journal of Lightwave Technology, vol. 23, no. 2, pp. 688-702, February 2005

7. R. Cardillo, V. Curri, M. Mellia: Considering Transmission Impairments in Wavelength Routed Networks, Optical Network Design and Models - ONDM 2005, February 7-9

8. Y. Huang, J. P. Heritage, and B. Mukherjee, Connection Provisioning with Transmission Impairment Consideration in Optical WDM Networks with High-Speed Channels, IEEE/OSA Journal of Lightwave Technology, vol. 23, no.3, March 2005

9. R. Martínez, C. Pinart, F. Cugini, N. Andriolli, L. Valcarenghi, P. Castoldi, L. Wosinska, J. Comellas and G. Junyent: Challenges and Requirements for Introducing ImpairmentAwareness into the Management and Control Planes of ASON/GMPLS WDM Networks, IEEE Communications Magazine, Vol. 44, No. 12, December 2006, pp76-85.

10. J. Strand, A. Chiu and R. Tkach, Issues for Routing in the Optical Layer, IEEE Communication Magazine, February 2001

11. Shu Shen, Gaoxi Xiao and Tee Hiang Cheng, The Performance of Periodic Link-State Update in Wavelength-Routed Networks, Broadnets 2006

12. F. Cugini, N. Andriolli, L. Valcarenghi, P. Castoldi, A Novel Signaling Approach to Encompass Physical Impairments in GMPLS Networks, IEEE Globecom 2004, pp369-373

13. GMPLS Lightwave Agile Switching Simulator (GLASS) http://snad.ncsl.nist.gov/glass/

14. AT\&T network topology: http://www.ssfnet.org/Exchange/gallery/usa/index.html

\section{Appendix A: Transmission Impairments models}

The transmission impairments considered in this paper include: Loss, ASE noise, PMD, CD, and Crosstalk. The final Signal Power and optical signal noise ratio (OSNR) will be checked at the end node. If the power and OSNR of signal at the end node can be accepted by the destination transponder, then the lightpath can be established.

By assuming an optical path is composed of $M$ fiber links, $M+1$ optical nodes and $N$ optical amplifiers, for a specific wavelength $m$,

- The final optical power can be modeled as $[4,5]$ :

$P_{\text {out }-m}=P_{\text {in }-m}+\sum_{j=1}^{N} G_{j}-\sum_{i=1}^{M} \operatorname{Loss}_{\text {fiber }-i} \cdot L_{i}-\sum_{k=1}^{M+1} \operatorname{Loss}_{\text {node }-k}$

where: $P_{\text {out }-m}$ is the final output power $(\mathrm{dBm})$ of wavelength $m ; P_{i n-m}$ is the input power $(\mathrm{dBm})$ of wavelength $m ; G_{j}$ is the gain of the $j^{\text {th }}$ optical amplifier. Loss fiber-i $_{\text {is }}$ is 
the $i^{\text {th }}$ fiber attenuation $(\mathrm{dB} / \mathrm{Km}) ; L_{i}$ is the $i^{\text {th }}$ fiber length $(\mathrm{Km}) ;$ Loss $_{\text {node- } k}$ is the power loss $(\mathrm{dB})$ for the specific switching path (add, drop or express) at the $k^{\text {th }}$ node.

- The optical OSNR is degraded by ASE noise in each optical amplifier [4]:

$O S N R_{\text {out }-m}=10 \cdot \log _{10}\left(\frac{1}{O S N R_{\text {in-m }}}+\frac{N F_{j} \cdot h v_{m} B}{P_{\text {in-m }}}\right)^{-1}$

where: $O S N R_{\text {out } m}$ is output OSNR (dB) of wavelength $m$ due to ASE noise in the $j^{\text {th }}$ amplifier; $O S N R_{i n-m}$ is the input channel OSNR (dB); $h$ is the Planck constant; $v_{m}$ is the frequency of wavelength $m ; B$ is the bandwidth of optical filter; $N F_{j}$ is the noise figure of the $j^{\text {th }}$ amplifier.

The optical OSNR is also degraded by accumulated CD, PMD and Cross-talk, which can be modeled as:

- $\quad$ The final CD [4]:

$C D_{\text {out }-m}=C D_{\text {in }-m}+\sum_{i}^{M}\left(C D_{\text {coeff }-i}+\Delta \lambda_{i} \times C D_{\text {slope }-i}\right) \times L_{i}-\sum_{j=1}^{N} D C_{d c u-j}$

where: $C D_{\text {out } m}$ is the final output $\mathrm{CD}(\mathrm{ps} / \mathrm{nm})$ of wavelength $m ; C D_{\text {in-m }}$ is the input $\mathrm{CD}$ (ps/nm); $C D_{\text {coeff- } i}$ is the CD coefficient for reference wavelength $(\mathrm{ps} / \mathrm{nm} / \mathrm{Km})$ in $i^{\text {th }}$ fiber; $C D_{\text {slope- } i}$ is the $C D$ slope coefficient $\left(\mathrm{ps} / \mathrm{nm}^{\wedge} 2 \mathrm{Km}\right)$ in $i^{\text {th }}$ fiber; $\Delta \lambda_{i}$ is the lambda deviation of wavelength $m$ from reference wavelength $(\mathrm{nm})$ in $i^{\text {th }}$ fiber; $D C_{d c u-j}$ is the dispersion compensation unit (DCU) module compensation value $(\mathrm{ps} / \mathrm{nm})$ in $j^{\text {th }}$ optical amplifier (we assume that DCU units are only located in the optical amplifiers)

- $\quad$ The final PMD [4]:

$$
P M D_{\text {out }-m}^{2}=P M D_{\text {in }-m}^{2}+\sum_{i}^{M} P M D_{\text {coeff }-i}^{2} \times L_{i}+\sum_{j=1}^{N}\left(P M D_{D C U-j}^{2}+P M D_{A M P-j}^{2}\right)+\sum_{k=1}^{M+1} P M D_{n o d e-k}^{2}
$$

where: $P M D_{\text {out }-m}$ is the output PMD (ps) of wavelength $m ; P M D_{f-\text { in }}$ is the input PMD (ps) of wavelength $m ; P M D_{\text {coeff }-i}$ is the PMD coefficient $(\mathrm{ps} / \sqrt{\mathrm{Km}})$ of the $i^{\text {th }}$ fiber ; $L_{i}$ is the $i^{\text {th }}$ fiber length $(\mathrm{Km}) ; P M D_{D C U-j}$ is the DCU module PMD value (ps) in $j^{\text {th }}$ amplifier; $P M D_{A M P-j}$ is the $j^{\text {th }}$ Amplifier PMD value (ps) ; $P M D_{W X C-k}$ is the PMD value (ps) of the $k^{\text {th }}$ optical node.

- The output Cross-talk after each node [4-6]:

$$
X t_{\text {out }-m}=10 \lg \left(10^{\frac{X t_{\text {in-m }}}{10}}+10^{\frac{X t_{\text {node }-k}}{10}}\right)
$$

where: $X t_{\text {out }-m}$ is the output crosstalk $(\mathrm{dBm}) ; X t_{\text {in-m }}$ is the input crosstalk $(\mathrm{dBm})$; $X t_{\text {node- } k}$ is the crosstalk value associated to $k^{\text {th }}$ node $(\mathrm{dBm})$.

In the destination node, the OSNR penalties due to CD, PMD, Crosstalk are calculated. Note that the transmission parameter values used in our simulations have not been included in this paper for space reason mainly; however, as a general indication, we have been assuming that all network links are based on Single Mode Fiber (SMF) and the optical amplifiers considered are based on EDFA (ErbiumDoped Fiber Amplifier) technology and compensated through DCF (Dispersion Compensating Fiber) units. 